\title{
Characterization of Low-Cost Materials as Human Tissue Equivalent Materials
}

\author{
Amirah Afiqah Bakri ${ }^{*}$ and Nik Noor Ashikin Nik Ab Razak ${ }^{2}$ \\ 1,2 School of Physics, Universiti Sains Malaysia, 18000, Pulau Pinang, Malaysia \\ *Corresponding author's email: (amirahafiqahh@gmail.com), Nik Noor Ashikin Nik Ab Razak()
}

\begin{abstract}
A phantom is a mass of material which is similar to human tissue. It is used to investigate the effect of radiation beams on human beings and also to simulate some form of human organs or tissues such as bone, muscles and kidney. Phantom materials can range from water to complex chemical mixtures that faithfully mimic the human body as it would interact with radiation. In this study, four tissue equivalent materials (Vaseline, cork, white cement, and paraffin wax) were characterised through their attenuation coefficients (linear and mass attenuation coefficients), and mass density. Their attenuation coefficients were analysed using Energy Dispersive Spectroscopy technique (EDS) with an Americium-241 source. The experimental results were compared with theoretical results from the International Commission on Radiation Units and Measurements, Report 44 (1989). This study proves that the lung-equivalent, brain matter-equivalent and bone-equivalent would be adequate to simulate lung, brain matter and bone tissue respectively.
\end{abstract}

Keywords - Phantom, tissue equivalent materials, attenuation coefficients, mass density.

\section{INTRODUCTION}

The selection of appropriate materials is crucial in the design and function of any type of phantom. A phantom is a mass of material which is similar to human tissue. It is used to investigate the effect of radiation beams on human beings and also to simulate some form of human organs or tissues such as bone, muscles and kidney. Phantom materials can range from water to complex chemical mixtures that faithfully mimic the human body as it would interact with radiation. A material which can be considered as tissue equivalent, it must have similar radiological properties as that of tissue (soft-tissue, muscle, bone, or fat). These properties include mass density, $\rho$, relative electron density and effective atomic number, $Z_{\text {eff }}$ as well as similar absorption and scattering of radiation [1]. Materials which are rich in carbon, calcium, hydrogen and oxygen were the most considered [2]. As for the image quality evaluation in diagnostic radiology, the ICRU Report 44 (1989) [3] recommends that tissue equivalent materials should have linear attenuation coefficients within $\pm 5 \%$ of these for a given body tissue over the required photon energy interval. Furthermore, it would be expected that their scattering properties could also be similar to those of human tissues [4]. An ideal tissue equivalent material would have similar properties of that tissue is simulated.

There are several findings which emphasize the characterization of the tissue equivalent materials through their attenuation properties. [5] used a polyenergetic X-ray beams at the energy range 12 to $54 \mathrm{keV}$ to determine the linear attenuation coefficient of several tissue equivalent materials used in CT (water, PMMA). A collimated beam of fluorescent x-rays with energies 9.88-59.32 keV were used to measure the linear attenuation coefficient of six types of human tissues and several tissue-equivalent materials, including water, nylon and polycarbonate [6]. A pulse-height spectroscopic technique was utilised to analyse the linear attenuation coefficients of commercially available composite phantom materials which designed to simulate the attenuation characteristics of breast fat and breast glandular tissue. This technique used a polyenergetic X-ray beams with energies varies from 18 to $100 \mathrm{keV}$ [7]. In this research study [8], it employed ${ }^{99 \mathrm{~m}} \mathrm{Tc}$ radionuclide source and a nuclear gamma camera as detector to analyse the water equivalence of solid phantoms. In this study [8], the water and four solid phantoms (RMI457, Plastic Water, RW3, and Perspex) were characterised through their attenuation properties. [9] the linear attenuation coefficient of low atomic polymer materials were measured using fluorescence $\mathrm{x}$-ray of energies 32 to $66 \mathrm{keV}$ and gamma rays of $140 \mathrm{keV}$ which were obtained from an unsealed ${ }^{99 \mathrm{~m}} \mathrm{Tc}$ source. In this work [10], investigated the suitability of the hydrophilic materials as tissue-equivalent in terms of their attenuation coefficient, which was measured using gamma-ray photons of energy $59.5 \mathrm{keV}$ and $\mathrm{x}$-ray beams of energy 17.44 and $44.23 \mathrm{keV}$. 
In this present study, the simultaneous measurement of the transmitted and scattered energy distribution technique was used to characterise four tissue equivalent materials (Vaseline, white cement, paraffin wax, and cork) through their attenuation properties.

\section{MATERIALS AND METHODOLOGY}

\subsection{Tissue Equivalent Samples}

In this project four tissue equivalent materials, brain matter-equivalent material (Vaseline), lung-equivalent material (cork), bone-equivalent material (white cement), and adipose-equivalent material (paraffin wax) were characterised, through their attenuation coefficients (linear and mass attenuation coefficients), and mass density. Table 1 shows the mass density of the tissue-equivalent materials reference values obtained from ICRU Report 44. The tissue equivalent materials sample, were measured inside a cuvette container with volume of $3.5 \mathrm{ml}$. The dimensions were chosen in order to provide sufficient single scattering events while minimizing the probability of multiple scattering and also reduce the uncertainties on the linear attenuation coefficient measurements [11].

\subsection{Experimental Set-up}

The experimental geometry in the present study is shown in Figure 1. The $59.5 \mathrm{keV} \gamma$-rays were emitted from a filtered low-energy photon point source of Am-241. The intensity of the source is $100 \mathrm{mCi}$. The EDS set-up consisted of an Am-241 with energy peak of $59.54 \mathrm{keV}$, a Sodium Iodide (NaI) detector, positioned at 0 degree with relation to the incident beam, used for detecting the energy distribution of transmitted beam (with the sample) or the incident beam (without the sample). The NaI detector was coupled to Multichannel Analyser. The monoenergetic beam were collimated to a narrow pencil beam, the sample is placed perpendicularly to the incident beam and the intensity of transmitted and incident beam are measured. The narrow beam is first projected without the sample as the background reading and the initial intensity is recorded. Then, the sample is placed perpendicularly to the incident beam, and attenuated intensity is recorded. The intensities of $\gamma$-rays were measured using a high-resolution NaI detector and the data were collected into the multichannel analyser. Acquisition times approximately $1000 \mathrm{~s}$ were used to achieve adequate counting statistics. The net counts without absorber $\left(I_{o}\right)$ and with absorber $(I)$ were obtained at the same experimental conditions. Linear attenuation coefficient $(\mu)$ is the fraction of attenuated incident photons per unit thickness of a material. It represents the fraction of photons removed from a monoenergetic beam per unit thickness of material. It is expressed numerically in units of $1 / \mathrm{cm}$. The linear attenuation coefficient of a material can be measured experimentally using the application of Beer-Lambert's law with standard transmission method by adopting narrow beam geometry. As seen in Figure 1, a parallel beam of monoenergetic photons passing through sample is attenuated due to absorption and scattering. Attenuation due to absorption follows the Beer-Lambert's rule

$$
\mathrm{I}=\mathrm{I}_{\mathrm{o}} \mathrm{e}^{-\mu \mathrm{t}}
$$

where $I_{0}$ is the incident $\gamma$-ray intensity when measured without sample, $I$ is $\gamma$-rays intensity transmitted through the sample, and $t$ is the sample thickness $(\mathrm{cm})$.

The experimental mass-absorption coefficient $(\mu / \rho)$ of elements is given by

$$
\mu / \rho=1 / \rho t . \ln \left(I_{o} / I\right)
$$

where $\rho$ is the material density $\left(\mathrm{g} / \mathrm{cm}^{3}\right)$. 


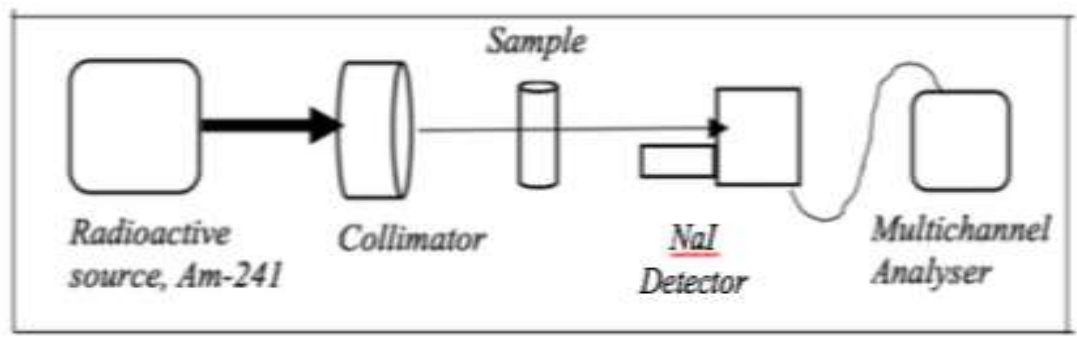

Figure 1: Schematic diagram for experiment set-up

\section{RESULTS AND DISCUSSION}

\subsection{Mass Density}

Table 1 shows the results of the mass density of the materials used in this research. It also showed the percentage difference of each material in relation to ICRU's human tissue which are being simulated. The mass density obtained for each material indicated a good concordance with respect to the mass density of those for human tissues which they simulate. The major absolute value of the percentage difference was $20.83 \%$ for white cement which is used as substitution of human bone. Even though the density value of white cement is high compared to ICRU, but its density is approximate to the density of compact bone. When compared to the attenuation value, the white cement shows a good agreement with the bone attenuation value which is very crucial when defining the tissue equivalent material.

Table 1: Density of four different tissue equivalent materials

\begin{tabular}{cccc}
\hline \multirow{2}{*}{ Material } & \multicolumn{2}{c}{ Density, $\boldsymbol{\rho}\left(\mathbf{g} / \mathbf{c m}^{\mathbf{3}}\right)$} & \\
\cline { 2 - 3 } & $\begin{array}{c}\text { Reference } \\
(\text { ICRU-44) }\end{array}$ & Experimental & \\
\hline Cork & $0.25-0.35$ & 0.30 & $3.33 \%$ \\
Vaseline & 1.04 & 0.93 & $10.58 \%$ \\
Paraffin Wax & 0.95 & 0.93 & $2.11 \%$ \\
White Cement & 1.92 & 2.32 & $20.83 \%$ \\
\hline
\end{tabular}

\subsection{Linear Attenuation Coefficient}

Linear attenuation coefficient $(\mu)$ is the fraction of attenuated incident photons per unit thickness of a material. It represents the fraction of photons removed from a monoenergetic beam per unit thickness of material. It is expressed numerically in units of $1 / \mathrm{cm}$. The linear attenuation coefficient of a material can be measured experimentally using the application of Beer-Lambert's law with standard transmission method by adopting narrow beam geometry. Table 2 shows the experimental linear attenuation coefficient for the tissue-equivalent materials studied in this research together with the theoretical values for human tissues. The percentage difference calculated for equivalent materials used in this study are within $\pm 5 \%$ which make the materials chosen in this project are ideal tissue equivalent material. Although the mass density of the white cement exhibited a deviation as a human bone substitute, it did present a good agreement in the total linear attenuation coefficient, which is the more indicated quantity for dosimetric comparisons. 
Table 2: Linear attenuation coefficient of various tissue equivalent materials

\begin{tabular}{cccc}
\hline \multirow{2}{*}{ Material } & \multicolumn{2}{c}{ Linear Attenuation Coefficient, $\boldsymbol{\mu}\left(\mathbf{c m}^{-\mathbf{1}}\right)$} & Percentage \\
\cline { 2 - 3 } & $\begin{array}{c}\text { Reference } \\
(\text { ICRU-44) }\end{array}$ & Experimental & Difference (\%) \\
\hline Cork & 0.1237 & 0.1258 & $1.60 \%$ \\
Vaseline & 0.2170 & 0.2136 & $1.54 \%$ \\
Paraffin Wax & 0.1872 & 0.1992 & $6.41 \%$ \\
White Cement & 0.6044 & 0.4855 & $5.71 \%$ \\
\hline
\end{tabular}

The linear attenuation coefficient properties obtained in this study, has validated that those tissue equivalent materials can be used to simulate human tissues. Among the commercialized materials studied in this work the best simulator for each tissue, considering the ICRU recommendations [3], are: cork, Vaseline and white cement for lung, brain matter and bone tissues, respectively. The results obtained for their linear attenuation coefficients showed differences of $5 \%$ with respect to the linear attenuation coefficients for biological tissues.

\section{CONCLUSION}

Based on the presented results, it can be concluded that a careful analysis in the choice of the most suitable tissue equivalent material is necessary and essential to simulate the attenuation properties of the human tissues, since these properties of commercial tissue equivalent material are very different. In order to characterize tissue equivalent materials, two parameters were used to compare with human tissue: (i) the experimental linear attenuation coefficient; and (ii) the experimental mass density. In this preliminary investigation, the percentage difference obtained for these two parameters were varied from $1 \%-6 \%$, and $0 \%-20 \%$, respectively. A more reasonable way for evaluating tissue equivalent materials is the total linear attenuation coefficient. The total linear attenuation coefficients results indicated that the Vaseline, white cement, and cork could be used as tissue equivalent according to the human tissue which they simulate. Therefore, the utilization of these materials for fabricating human phantoms are good and low-cost alternative.

\section{ACKNOWLEDGEMENT}

We thank Universiti Sains Malaysia for providing us Research University Grant 1001/PFIZIK/8011087 to support this work.

\section{REFERENCES}

[1] White, D. R. (1978). Tissue substitutes in experimental radiation physics. Medical Physics, 5(6), 467-479.

[2] Claude, K. P., Tagoe, S. N., Schandorf, C., \& Amuasi, J. (n.d.). Fabrication of a tissue characterization phantom from indigenous materials for computed tomography electron density calibration. Retrieved from https://sar.org.za/index.php/sar/article/view/237

[3] ICRU, Report44. (1989). Tissue Substitutes in Radiation Dosimetry and Measurement Bethesda.

[4] Poletti, M. E., Gonçalves, O. D., \& Mazzaro, I. (2001). X-ray scattering from human breast tissues and breastequivalent materials. Physics in Medicine and Biology, 47(1), 47-63.

[5] Ferreira, C., Filho, R. X., Vieira, J., Tomal, A., Poletti, M., Garcia, C., \& Maia, A. (2010). Evaluation of tissueequivalent materials to be used as human brain tissue substitute in dosimetry for diagnostic radiology. Nuclear Instruments and Methods in Physics Research Section B: Beam Interactions with Materials and Atoms,268(16), 2515-2521. doi:10.1016/j.nimb.2010.05.051

[6] White, D. R., Peaple, L. H., \& Crosby, T. J. (1980). Measured Attenuation Coefficients at Low Photon Energies (9.88-59.32 keV) for 44 Materials and Tissues. Radiation Research, 84(2), 239. doi:10.2307/3575295

[7] J.W.Byng, J.G.Mainprize, and M.J.Yaffe. (1998). X-ray characterization of breast phantom materials. Phys. Med. Biol., vol. 43, pp. 1367-1377.

[8] Hill, R., Brown, S., \& Baldock, C. (2008). Evaluation of the water equivalence of solid phantoms using gamma ray transmission measurements. Radiation $\quad$ Measurements, 43(7), doi:10.1016/j.radmeas.2008.01.019

[9] Midgley, S. (2005). Measurements of the X-ray linear attenuation coefficient for low atomic number materials at energies 32-66 and. Radiation Physics and Chemistry, 72(4), 525-535. doi:10.1016/j.radphyschem.2004.02.001 
[10] Farquharson, M., Spyrou, N., Al-Bahri, J., \& Highgate, D. (1995). Low energy photon attenuation measurements of hydrophilic materials for tissue equivalent phantoms. Applied Radiation and Isotopes, 46(8), 783-790. doi:10.1016/0969-8043(95)00025-9

[11] Poletti, M., Gonçalves, O., Schechter, H., \& Mazzaro, I. (2002). Precise evaluation of elastic differential scattering cross-sections and their uncertainties in X-ray scattering experiments. Nuclear Instruments and Methods in Physics Research Section B: Beam Interactions with Materials and Atoms, 187(4), 437-446. doi:10.1016/s0168-583x(01)01149-1 